\title{
Diversity As a Selection Pressure in Dynamic Environments
}

\author{
Lam T. Bui \\ School of ITEE, University of \\ New South Wales@ ADFA \\ NorthCott Drive, Canberra \\ ACT, Australia, 2600 \\ I.bui@adfa.edu.au
}

\author{
Jürgen Branke \\ Institute AIFB, University of \\ Karlsruhe \\ 76128 Karlsruhe Germany \\ branke@aifb.uni- \\ karlsruhe.de
}

\author{
Hussein A. Abbass \\ School of ITEE, University of \\ New South Wales@ ADFA \\ NorthCott Drive, Canberra \\ ACT, Australia, 2600 \\ a.abbass@adfa.edu.au
}

\begin{abstract}
Evolutionary algorithms (EAs) are widely used to deal with optimization problems in dynamic environments (DE) [3]. When using EAs to solve DE problems, we are usually interested in the algorithm's ability to adapt and recover from the changes. One of the main problems facing an evolutionary method when solving DE problems is the loss of genetic diversity.

In this paper, we investigate the use of evolutionary multiobjective optimization methods (EMOs) for single-objective DE problems. For that purpose, we introduce an artificial second objective with the aim to maintain useful diversity in the population. Six different artificial objectives are examined and compared.

All the results will be compared against a traditional GA and the random immigrants algorithm[4]. NSGA2 is employed as the evolutionary multi-objective technique.
\end{abstract}

Categories and Subject Descriptors: B.X.X [Genetic algorithms]:

General Terms: Algorithms, Performance.

Keywords: Dynamic environments, diversity, random immigrants, multi-objective.

\section{METHODS}

The idea of using EMOs to solve single objective optimization problems has been proposed before, see e.g. [7, 6, $2]$. There, the idea was to use additional auxiliary objectives to guide the search and improve the algorithm's search capabilities. Yamasaki [9] introduced a time stemp as a second objective with the aim to maintain useful diversity in a dynamic environment. Diversity as additional objective has been explicitly considered in [8], but for static optimization problems.

We extend on the above ideas by testing six different auxiliary objectives to maintain useful diversity in a dynamic environment: The first artificial objective is a time stamp of

Copyright is held by the author/owner.

GECCO'05, June 25-29, 2005, Washington, DC, USA.

ACM 1-59593-010-8/05/0006. when an individual is generated. As in [2], we stamp each individual in the initial population with a different time stamp represented by a counter that gets incremented every time a new individual is created. From the second population, all individuals in the population get the same time stamp that is set to the population size plus the generation index. The second artificial objective is simply to assign each individual a random value. Some bad individuals may be assigned smaller random values and get a chance to survive; hence they may deem to be useful when the environment changes. The third approach invert the original objective function by minimizing it if it was a maximization problem, and vice-versa. The last three options are determined based on the Euclidean distance: the distance to the closet neighbor (DCN), the average distance to all individuals (ADI) and the distance to the best individual of the population (DBI), all of them to be maximized. Note that among these options, DCN and ADI take much more time to calculate the distances than DBI.

\section{EXPERIMENTAL SETTINGS}

For testing, we use the Moving Peaks Benchmark (MPB) intorudce by Branke [5]. The MPB is a dynamic benchmark problem with a number of peaks changing over time in location, width, and height. The details of the problem and its settings can be found in [1]. We selected a similar scenario as Branke's second scenario with 50 peaks, 5 dimensions, change frequency of 35 generations, height and width change severities of 7.0 and 1.0 and change distance of 1.0. The traditional GA uses binary tournament selection, single point crossover and bit-flip mutation. The random immigrants algorithm replaces $20 \%$ of the population at each generation with new individuals. In order to have a fair comparison, we also employ elitism for both algorithms. The behavior of evolutionary methods would normally depend on the crossover and mutation rates used. Therefore, there is a need to examine the different methods with a wide range of parameter values to identify a good setting. The crossover rate $p c$ is varied between 0.5 and 1 with a step of 0.05 and the mutation rate $p m$ is varied between 0 and 0.2 with a step of 0.01 . For each pair of $p c$ and $p m$, thirty runs are performed with different random seeds, a population size of 100 , the number of generations is 1000 , and chromosomes are binary encoded with length 150 bits. Identical settings are also used for NSGA2.

We record the best individual in each generation as measured on the original single objective function. The dif- 
ference between the objective value of this individual and the current global optima is known as the generation error (GEr). We recorded the generation error and derived the average generation error of each last generation just before a new change occurs (AGEr).

\section{RESULTS AND DISCUSSION}

We generate the averaged generation error AGEr for different values of crossover and mutation for each artificial objective as well as its variance. An example is given in Figure 1. It is clear that the absence of mutation deteriorates the quality of solutions. For EMO approaches, in general, a good performance was achieved within an area centered on $(0.6,0.07)$. In this area of the crossover and mutation space, the DCN and ADI approaches produced the best results. The traditional GA and random immigrants are not only clearly inferior, but also very sensitive to the mutation rate (see Figure 1). The best mutation rate which achieved the minimum AGEr is 0.04 for the traditional GA and 0.01 for the random immigrants.

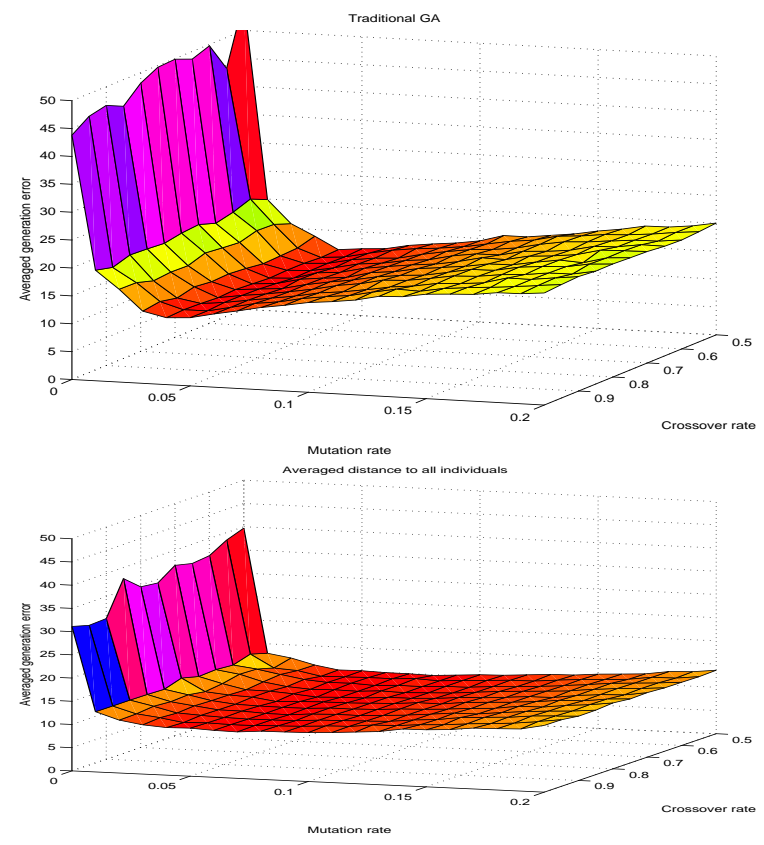

Figure 1: The AGEr achieved over different ranges of $p c$ and $p m$ for the standard EA (top) and the ADI approach (bottom).

Overall, each approach had a different crossover and mutation pair of values, where the best performance was achieved. Table 1 summarizes these results. It is interesting to note that the traditional GA approach achieved very similar results as the random immigrants approach. The small mutation rate for the random immigrants approach appears to be due to the fact that diversity is already maintained through the introduction of new children at random. Therefore, the mutation probability did not have much impact on the quality of obtained solutions.
Table 1: The best $p c$ and $p m$ for each approach and the AGEr \pm the standard error

\begin{tabular}{lcc}
\hline $\begin{array}{l}\text { The artificial } \\
\text { objective function }\end{array}$ & $\begin{array}{c}\text { Crossover (Mutation) } \\
\text { Probabilities }\end{array}$ & AGEr \\
\hline Traditional GA & $0.55(0.04)$ & $11.48 \pm 0.60$ \\
Random Immigrants & $1.00(0.01)$ & $11.47 \pm 0.56$ \\
Time-based objective & $0.60(0.11)$ & $12.06 \pm 0.64$ \\
Random objective & $0.60(0.10)$ & $11.29 \pm 0.55$ \\
Inverse objective & $0.55(0.06)$ & $12.37 \pm 0.87$ \\
DCN & $0.75(0.04)$ & $9.52 \pm 0.45$ \\
ADI & $0.70(0.06)$ & $9.74 \pm 0.35$ \\
DBI & $0.50(0.09)$ & $12.24 \pm 0.55$ \\
\hline
\end{tabular}

\section{CONCLUSION}

In this paper, we examined the feasibility of applying EMOs in dynamic environments. In order to do that, we selected NSGA2 and the moving-peaks benchmark. The traditional GA, random immigrants and six approaches (EMO approaches) for defining artificial objectives for the problem were employed: time-based, random, objective reverse, distance to the closet neighbor, the averaged distance to all other individuals, and the distance to the best individual. As a result of the experiments, EMO approaches showed competitive performance. Within EMO approaches, the averaged distance to all other individuals, and the distance to the nearest neighbor options have significantly outperformed the other approaches.

\section{ACKNOWLEDGMENTS}

This work is supported by the University of New South Wales grant PS04411 and the Australian Research Council (ARC) Centre on Complex Systems grant number CEO0348249.

\section{REFERENCES}

[1] Online. http://www.aifb.uni-karlsruhe.de/ jbr/MovPeaks.

[2] H. A. Abbass and K. Deb. Searching under multi-evolutionary pressures. In Zitzler et al., editor, Proceedings of the Fourth Conference on Evolutionary Multi-Criterion Optimization, Spain, 2003.

[3] J. Branke. Evolutionary optimization in dynamic environments. Kluwer Academic Publishers, Massachusetts USA, 2002.

[4] J.J. Grefenstette. Genetic algorithms for changing environments. In R.Männer and B.Manderick, editors, Parallel Problem Solving from Nature, pages 137-144. Elsevier Science Publisher, 1992.

[5] J.Branke. Memory enhanced evolutionary algorithms for changing optimisation problems. In In Congress on Evolutionary Computation CEC99, pages 1875-1882. IEEE, 1999.

[6] M.T. Jensen. Helper-objectives: Using multiobjective evolutionary algorithms for single-objective optimization. Journal of Mathematical Modelling and Algorithms, 1(25), 2004.

[7] J. Knowles and D. Corne. Reducing local optima in single objective problems by multi-objectivization. In Zitzler et al., editor, Proceedings of the First Conference on Evolutionary Multi-Criterion Optimization, Zurich Switzeland, 2001.

[8] A. Toffolo and E. Benini. Genetic diversity as an objective in multi-objective evolutionary algorithms. Evolutionary Computation, 11(2):151-168, 2003.

[9] K. Yamasaki. Dynamic pareto optimum GA against the changing environments. In J. Branke and T. Bäck, editors, Evolutionary Algorithms for Dynamic Optimization Problems, pages 47-50, San Francisco, California, USA, 72001. 